\title{
Semiconductor parameter extraction via current-voltage characterization and Bayesian inference methods
}

\author{
Rachel C. Kurchin, Jeremy R. Poindexter, Daniil Kitchaev, Ville Vähänissi, Carlos del Cañizo, Liu \\ Zhe, Hannu S. Laine , Chris Roat, Sergiu Levcenco, Gerbrand Ceder , Tonio Buonassisi
}

\begin{abstract}
Defects in semiconductors, although atomistic in scale and often scarce in concentration, frequently represent the performance-limiting factor in optoelectronic devices such as solar cells. However, due to this scale and scarcity, direct experimental characterization of defects is technically challenging, timeconsuming, and expensive. Even so, the fact that defects can limit device performance suggests that device-level characterization should be able to lend insight into their properties. In this work, we use Bayesian inference to demonstrate a way to relate experimental device measurements with defect properties (as well as other materials properties affected by the presence of defects, such as minority-carrier lifetime). We apply this method to solve the "inverse problem" to a forward device model - namely, determining which input parameters to the model produce the measured electrical output. This approach has distinct advantages over direct characterization. First, a single set of measurements can be used to determine many parameters (the number of which, in principle, is limited only by the computing resources available), saving time and cost of facilities and equipment. Second, since measurements are performed on materials and interfaces in their relevant device geometries (vs. separately prepared samples), the determined parameters are guaranteed to be physically relevant. We demonstrate application of this method to both tin monosulfide and silicon solar cells and discuss potential for future application in a broader array of systems.
\end{abstract}

Index Terms - Bayes methods, charge carrier lifetime, charge carrier mobility, parameter estimation, photovoltaic cells, silicon.

\section{Defect Parameters in SEMICONDUCtors}

Defects in semiconductor devices frequently play a defining role in determining optoelectronic material and device properties, often even at trace concentrations (i.e., parts per billion or below). This is especially true for devices in which minority carriers determine operating mechanisms, such as photovoltaic solar cells and light-emitting diodes. Thus, in such devices, defect engineering remains an effort critical to ensuring high performance. Unfortunately, this effort is hampered by the fact that basic defect parameters are often unknown and difficult to measure, typically requiring specialized techniques (such as deep-level transient spectroscopy) and device architectures.

For minority-carrier devices in which Shockley-Read-Hall (SRH) recombination (also called defect-assisted recombination) dominates, the defect parameters of interest are defect density $\left(N_{*}\right)$, energy level $(E)$, and capture cross-sections

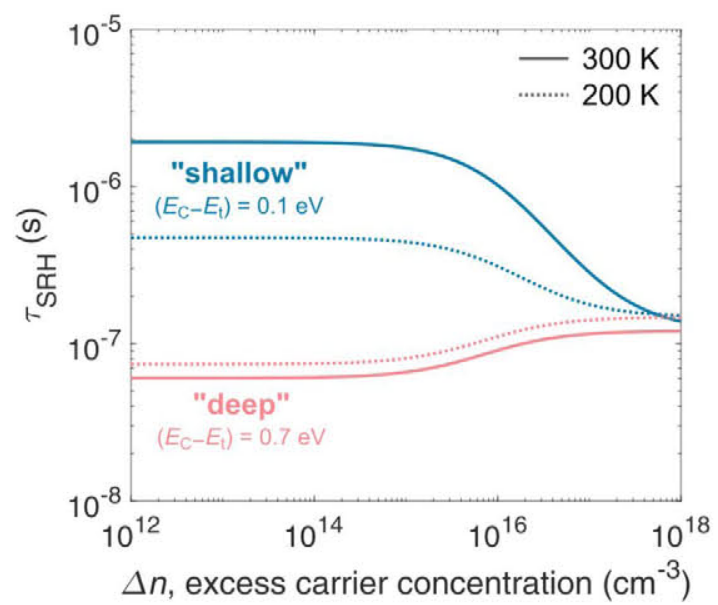

Fig. 1. Plot of Shockley-Read-Hall lifetime as a function of injection level in a "generic" hypothetical $p$-type semiconductor for "shallow" (blue lines) and "deep" (pink lines) defect levels at 300 and $200 \mathrm{~K}$. The modeled semiconductor has a band gap of $1.4 \mathrm{eV}, N$ $=10^{16} \mathrm{~cm}^{-3}, \sigma_{\mathrm{e}, \mathrm{h}}=10^{-16} \mathrm{~cm}^{2}$, densities of states equal to $10^{19} \mathrm{~cm}^{-3}$ for both the valence $\left(E_{\mathrm{V}}\right)$ and conduction band $\left(E_{\mathrm{C}}\right)$, acceptor density $=$ $10^{16} \mathrm{~cm}^{-3}$, and electron and hole effective masses both equal to 0.50 .

for electrons and holes $\left(\sigma_{* n}\right)[1,2]$. Through these parameters, the effects of both injection level and temperature can greatly alter the strength of recombination, resulting in different regimes of behavior. Figure 1 illustrates a simple example of these regimes in a "generic" hypothetical $p$-type semiconductor (see caption for details). The effect of "shallow" vs. "deep" traps have opposite effects on SRH lifetime $\left(\tau_{\text {sи }}\right)$ with respect to injection level-i.e., a significant decrease vs. slight increase (Figure 1, solid lines). Similarly, at lower temperatures $\tau_{\text {жี }}$ decreases for a shallow defect, whereas it increases for a deep defect (Figure 1, dotted lines). These behaviors are the basis for temperaturedependent and injection-dependent lifetime spectroscopy, which as documented by Rein et al. [3] can in theory be used to identify defects based on these divergent behaviors. In practice, however, the numerous possible combinations of $N_{t}, E_{\mathrm{s}}, \sigma_{\mathrm{s}}$, and $\sigma_{\mathrm{h}}$ can vastly complicate the process of finding confident fits to these parameters.

In this work, we aim to extract basic defect parameters in photovoltaic semiconductors by feeding experimental solar cell measurements (i.e., temperature- and illumination-dependent 
current-voltage data, or $J V T i$ ) into a numerical solar cell model developed in PC1D and applying a Bayesian inference algorithm. We first demonstrate the Bayesian inference approach by calculating probability distributions of materials and device parameters in SnS solar cells [4], some of which had not been previously measurable. We then outline our approach to applying this method to extract $E_{\mathrm{s}}, \sigma_{\mathrm{s}}$, and $\sigma_{\mathrm{n}}$ for interstitial iron $(\mathrm{Fe}$ ) in silicon solar cells. These results serve as validation for using this new method of defect characterization on newer, less understood semiconductor materials for which defect parameters are likely less well known.

\section{BAYESIAN INFERENCE ALGORITHM}

Bayes' Theorem is a statement about the relationship between conditional probabilities. With $H$ as a hypothesis and $E$ the evidence:

$$
P(H \mid E)=\frac{P(H) P(E \mid H)}{P(E)}
$$

$P(H)$ is referred to as the prior distribution, representing our initial beliefs about how a system will behave. Bayes' theorem gives us a prescription for how to update this prior when presented with some evidence $E$ (for example, the outcome of an experimental measurement) to create the posterior distribution $P(H \mid E)$. In particular, we use the likelihood $P(E \mid H)$, or the probability that we would observe the evidence if our hypothesis is true. We can perform this procedure iteratively to further update our posterior in the face of further measurements.

The power of this approach comes from the ability to fit many parameters, for which the parameter space and shape of the underlying probability distribution may be complicated. In such a case, a fitting approach such as a least-squares regression may fall into a "local minimum" and miss the true values of the materials properties to be measured. The Bayes approach instead produces a probability distribution over the entire parameter space, which yields not only the most likely values of parameters, but also insight into how parameters may depend upon one another.

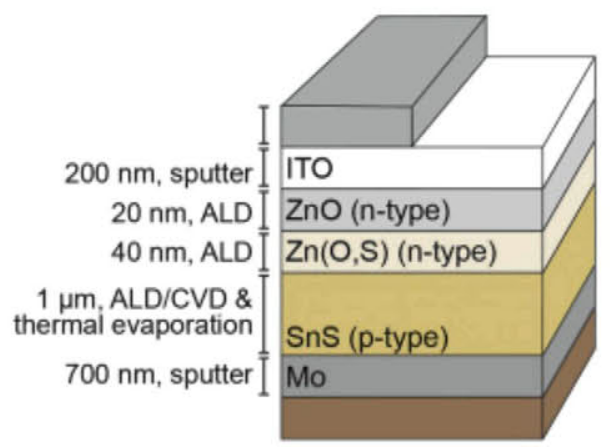

Fig. 2. Schematic (not to scale) of the SnS device that was characterized.

\section{JVTI MEASUREMENTS ON SNS SOLAR CELLS}

As a first proof of concept, we applied this approach to tin monosulfide (SnS) solar cells [5]. SnS is a relatively new candidate photovoltaic material, but one that our group had extensively characterized in the past, making it a fruitful platform to verify the Bayesian method. Using a collection of current-voltage curves taken at three different temperatures and two illumination levels, we generated a probability distribution over four "unknown" input parameters to a SCAPS-1D [6] model of the devices: minority carrier (electron) mobility $\mu$ and lifetime $\tau$ in the SnS absorber layer, and the conduction band offset $\Delta E_{\mathrm{C}}$ and surface recombination velocity $S_{\text {eff }}$ at the interface between the absorber and the $\mathrm{Zn}(\mathrm{O}, \mathrm{S})$ buffer layer. A schematic of the device is shown in Figure 2. The results of this analysis are shown in Figure 3. The conduction band offset was fit to be $-0.21 \pm 0.03 \mathrm{eV}$, consistent with the previous measurement of $-0.38 \pm 0.2 \mathrm{eV}$ [7] and fit with a higher precision than direct measurement allows.

While the lifetime and mobility did not individually converge in this analysis, the two-variable marginalization shows a line of higher probability corresponding to a constant product $\mu \tau$, indicating that the diffusion length is well-constrained by the measurement. Surface recombination velocity $S_{\text {eff }}$ had never previously been measured for this interface, and was fit to between $1,000-1,800 \mathrm{~cm} / \mathrm{s}$. Seff is an example of a property that is extremely difficult to measure but can be limiting to device performance, thus emphasizing the value of this Bayesian fitting technique in this scenario.

The inset in Figure 3 shows the Shannon entropy $(\Sigma P \log P)$ of each parameter individually as well as the overall

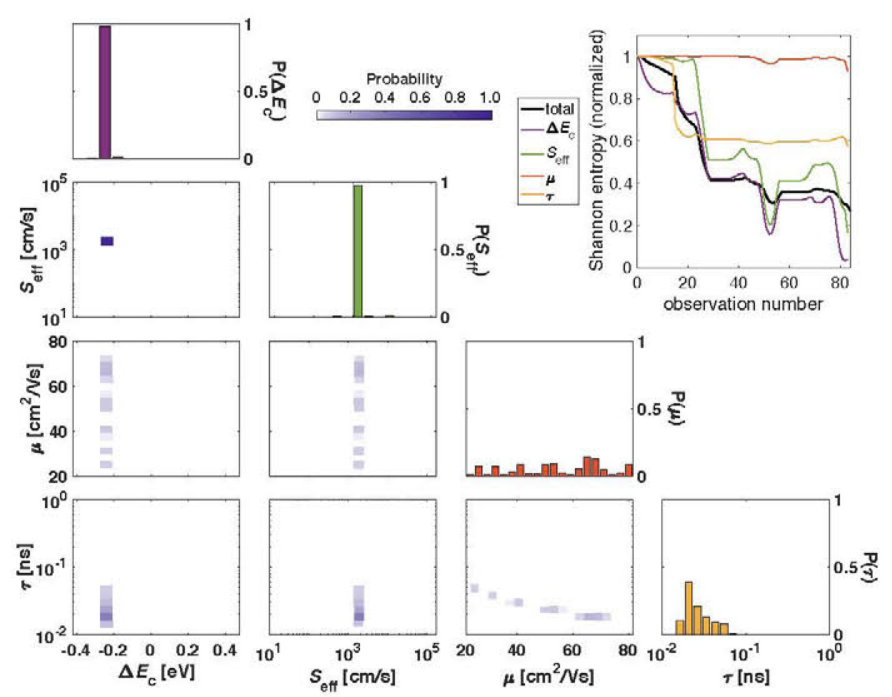

Fig. 3. Results of SnS device Bayesian analysis. On the diagonal are single-variable distributions over each of the four parameters, and below the diagonal the corresponding two-variable marginalizations. Inset shows normalized Shannon entropy as a function of number of observations fed into the inference routine. 
distribution, normalized to start at unity for the uniform distribution in each case. This is a further indication that the fit is converging as more data is fed into the Bayesian inference algorithm.

\section{JVTI MEASUREMENTS ON IRON-CONTAMINATED SILICON SOLAR CELLS}

Defect parameters such as energy level and capture crosssection are also, like $S_{\text {eff, }}$ physical parameters that are extremely difficult to probe directly. To further validate our methods, we choose to apply our framework to silicon solar cells contaminated with known levels of iron, an impurity whose defect parameters in silicon have been thoroughly characterized [8]. We utilize samples from a previous work by Vähänissi $e t$ al. [9] where the interstitial iron concentrations (after cell processing) have been measured at $N_{\mathrm{t}}=2 \times 10^{12} \mathrm{~cm}^{-3}$. This constrains an additional parameter, improving the quality of our fits to $E_{\mathrm{s}}, \sigma_{\mathrm{s}}$, and $\sigma_{\mathrm{s}}$.

$J V T i$ measurements were performed on an intentionally contaminated sample (initial $[\mathrm{Fe}]=2 \times 10^{4} \mathrm{~cm}^{-3}$ ) and an uncontaminated reference-the "60A" samples from Ref. [9]. Semi-automated current-voltage measurements were performed using a Keithley 2400 sourcemeter connected to LabView. Temperature was varied from 300 to $175 \mathrm{~K}$ (in increments of $25 \mathrm{~K}$ ) using an ARS cryostat (DE-204SI), liquid helium compressor (ARS-4HW), and polyimide heater (Minco HAP6943) under vacuum controlled by a LakeShore 331 temperature controller. Eight illumination levels were measured-at 1.01, 0.92, 0.69, 0.31, 0.093, 0.048, 0.0093, and 0.0045 Suns - using a Newport Oriel LCS-100 Solar Simulator and neutral density filters.

As a preliminary analysis, we used the Bayesian inference routine to fit a simple ideal diode model:

$$
J(V, T, i)=J_{0}(T, i)\left(e^{-\frac{q V}{n k T}}-1\right)+J_{\mathrm{sc}}
$$

where $J_{\circ}$ is given by[10]:

$$
J_{0}(T, i)=\frac{q n_{i}^{2}(T)}{N_{A}} \sqrt{\frac{D_{n}}{\tau_{n}(i)}}
$$

With only two free parameters (ideality factor $n$ and electron lifetime $\tau_{\text {s }}$ ) we were able to model the current-voltage curves relatively accurately and estimate fitted lifetimes corresponding to the orders of magnitude expected, as shown in Figure 4.

To obtain estimates for specific defect parameters $E_{\mathrm{l}}, \sigma_{\mathrm{s}}$, and $\sigma_{\mathrm{r}}$, we extend this analysis using a numerical model in cmd-PC1D 6.2 [9,10], which incorporates full Shockley-ReadHall recombination analysis with built-in dependencies on temperature and injection level. Results from these fits are shown in Figure 5, and JV curves corresponding to the highest-probability parameter values in Figure $6 . \tau_{\mathrm{n} 0}$ and $\tau_{\mathrm{n} 0}$ (along with $E_{\mathrm{t}}$ ) were the direct inputs, but they can be easily related to $\sigma$.

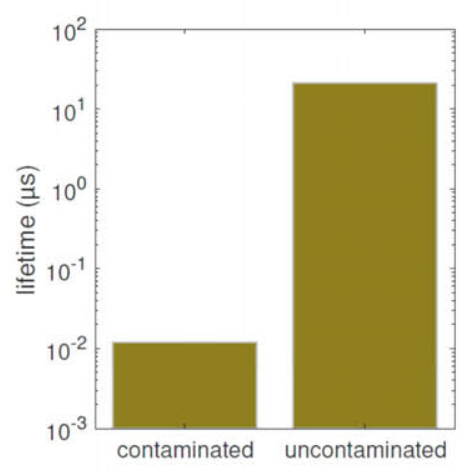

Fig. 4. Results of ideal diode equation fit to data on silicon devices both contaminated and uncontaminated with iron.
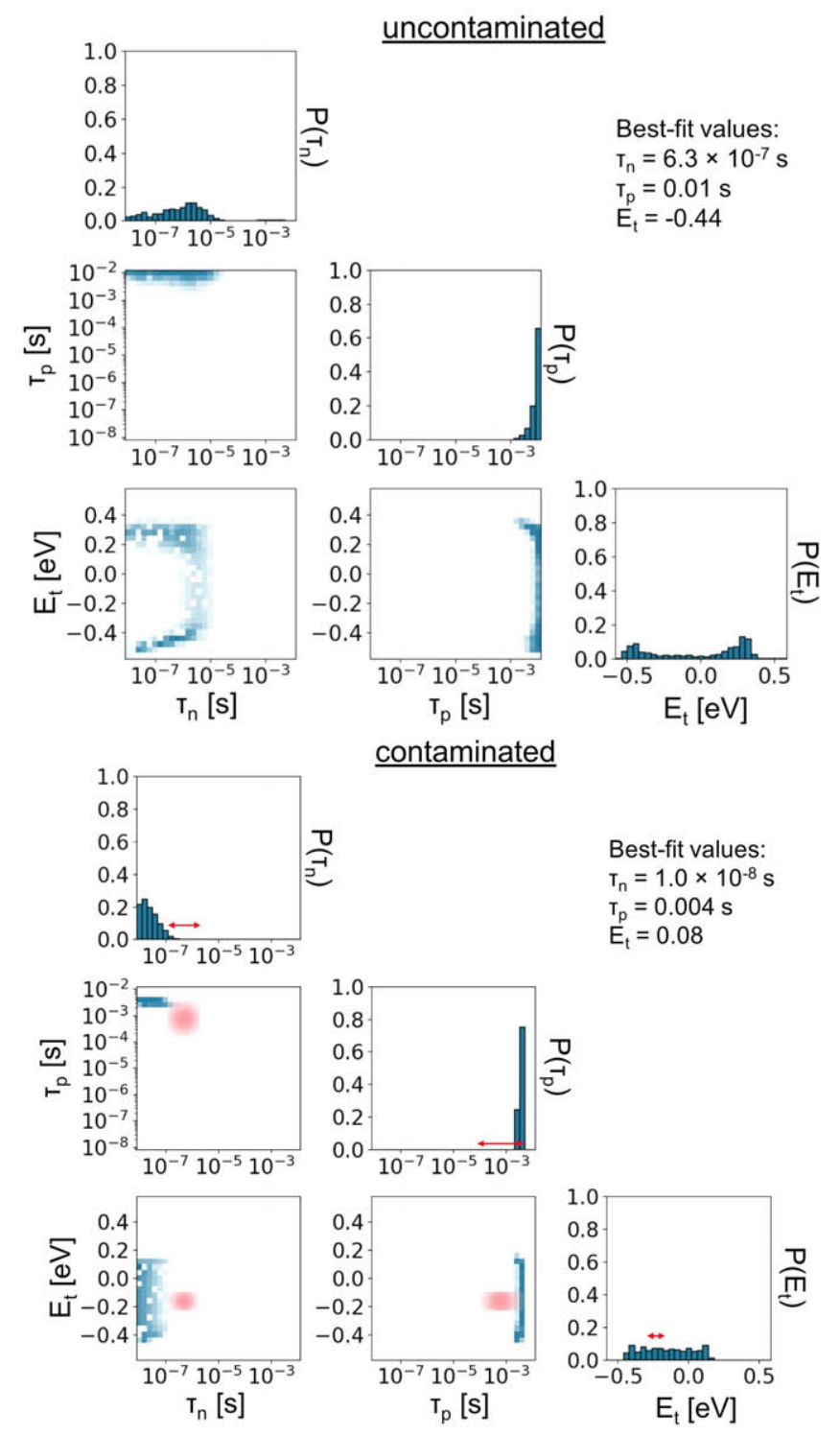

Fig. 5. Results of PC1D model Bayesian fits to uncontaminated (top) and contaminated (bottom) samples. Literature-reported values are overlaid in red for contaminated sample. 


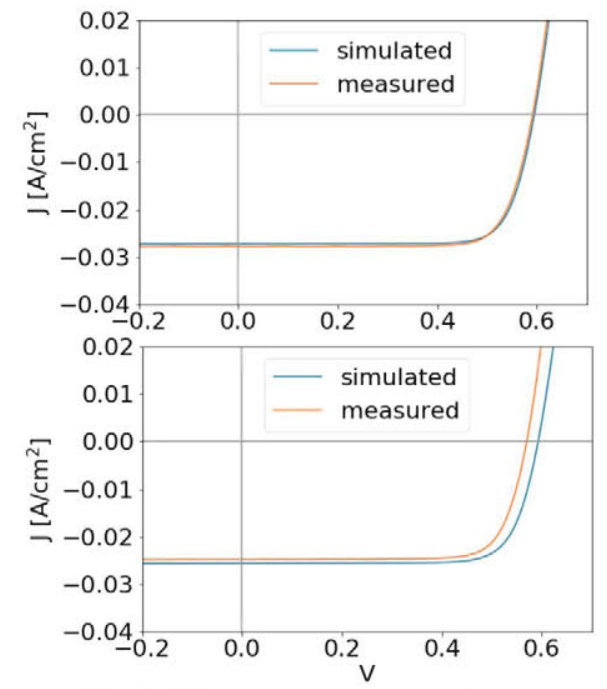

Fig 6. 1-Sun, 300K JV curves corresponding to the highestprobability parameter values for the uncontaminated (top) and contaminated (bottom) samples.

and $\sigma_{\mathrm{n}}$ (which are not directly accessible in PC1D) using the SRH equations. [1,2]

Bayesian inference results for the uncontaminated cell show values for $\tau_{\mathrm{n} 0}$ broadly distributed between approximately $10^{-7}-$ $10^{-5} \mathrm{~s}(100 \mathrm{~ns}-10 \mu \mathrm{s})$, along with values for $E_{\mathrm{t}}$ that peak at $-0.45 \mathrm{eV}$ and $+0.30 \mathrm{eV}$ compared to the intrinsic level $E_{\mathrm{i}}$ (which is $0.56 \mathrm{eV}$ above the valence band $E_{\mathrm{V}}$ ). When looking at the covariance of these two parameters (lower-left plot), it becomes clear that "constant recombination space" involves a tradeoff-higher values of $\tau_{\mathrm{n} 0}$ correlate with "deeper" traps $\left(E_{\mathrm{t}}\right.$ closer to zero). This behavior is expected from SRH recombination statistics. The high values of $\tau_{\mathrm{p} 0}$ are also expected for a $p$-type absorber-in other words, majoritycarrier recombination parameters do not limit recombination. It is also instructive to note that the values for $\tau_{\mathrm{n} 0}$ here are slightly lower than those extracted from Bayesian analysis with the ideal-diode framework in Figure 4.

Bayesian inference results for the contaminated cell show uniformly lower values for $\tau_{\mathrm{n} 0}$, peaking below $10^{-8} \mathrm{~s}$. Assuming a trap density $N_{\mathrm{t}}=2 \times 10^{12} \mathrm{~cm}^{-3}$ as previously measured directly [9] along with a thermal velocity $v_{\text {th,e }}=2.6 \times 10^{7} \mathrm{~cm} / \mathrm{s}$, a range of $\tau_{\mathrm{n} 0}$ between $10^{-8}-10^{-7} \mathrm{~s}$ corresponds

to a range of $\sigma_{\mathrm{e}}$ between $2 \times 10^{-12}-2 \times 10^{-13} \mathrm{~cm}^{2}$. The upper value in this range is slightly larger than the actual value of $4 \times 10^{-14} \mathrm{~cm}^{2}[6,11]$ which might be explained by an underestimate of $N_{\mathrm{t}}$ or the unaccounted-for presence of a different defect. Despite this slight discrepancy, it remains clear that the Bayesian inference clearly identified differences in values for $\tau_{\mathrm{n} 0}$ between the uncontaminated and contaminated samples.

Similarly, a value for $\tau_{\mathrm{p} 0}$ of $2 \times 10^{-3} \mathrm{~s}$, using the same value for $N_{\mathrm{t}}$ and a thermal velocity $v_{\mathrm{th}, \mathrm{h}}=1.7 \times 10^{7} \mathrm{~cm} / \mathrm{s}$, corresponds to a value of $\sigma_{\mathrm{h}}=1.5 \times 10^{-17} \mathrm{~cm}^{2}$, which falls well within the range of values reported in literature [8].

For $E_{\mathrm{t}}$, the values are less well converged without further subdivision within the Bayesian inference algorithm, which is planned as a continuation of this work. Still, they are relatively well centered around the actual value of $E_{\mathrm{i}}-0.185 \mathrm{eV}$ (or $E_{\mathrm{V}}+$ $0.375 \mathrm{eV})[6,12]$.

Overall, comparing the results between the uncontaminated and contaminated cells, this Bayesian inference framework clearly can distinguish high and low lifetimes, and helps show how defect parameters covary. Estimated values for $E_{\mathrm{t}}, \sigma_{\mathrm{e}}$, and $\sigma_{\mathrm{h}}$ all fall close to or within ranges documented in literature, which demonstrates general promise for applying Bayesian inference approaches to extract defect parameters in solar cells. We are optimistic that with further refinement to the models (e.g. including temperature dependence of capture crosssections as described in [8]) and updates to the computational tools (e.g. to allow more free parameters), we can improve the accuracy and precision of these fits and make the approach more suitable for application in less thoroughly characterized systems.

\section{CONCLUSIONS AND ACKNOWLEDGEMENTS}

Bayesian inference as a technique to invert numerical device models is a powerful approach enabling extraction of values for multiple arbitrary physical parameters from a set of simple, automatable, electrical measurements. This approach is preferable in many cases to directly measuring these parameters. It is significantly faster, requiring approximately a day each of measurement and compute time, assuming a modest ( 60 -core) high-performance cluster. It is also less expensive due to fewer equipment requirements. In addition, and perhaps most importantly, it can offer more physically relevant measurements of the parameters than conventional "direct" measurement techniques. For example, Hall mobility measurements probe lateral majority-carrier mobility, while through-film minority carrier mobility is the relevant limiting factor in PV devices. Especially in new materials being explored for PV, many of which exhibit anisotropic charge transport properties, this difference can be critical. The Bayesian approach, by using actual device data, by definition gives the device-relevant version of all such parameters. In our latest study of iron-contaminated silicon devices, we demonstrate that the approach can also be fruitful in measuring defect parameters, which we hope to apply to novel PV materials to accelerate identification of performance-limiting properties.

\section{ACKNOWLEDGEMENTS}

R. C. Kurchin and J. R. Poindexter contributed equally to this work. R. C. Kurchin acknowledges the support of a Blue Waters Graduate Fellowship and a MIT Energy Initiative Total Energy Fellowship. J. R. Poindexter acknowledges the support from the Martin Family Society of Fellows for Sustainability and the Switzer Environmental Fellowship. Simulations and analysis were performed using computational resources sponsored by the Department of Energy's Office of Energy Efficiency and Renewable Energy and located at the NREL. We also acknowledge funding support from a Google Faculty Research Grant. 


\section{REFERENCES}

[1] W. Shockley and W. T. Read, "Statistics of the Recombination of Holes and Electrons," Phys. Rev., vol. 87, no. 46, pp. 835-842, 1952.

[2] R. N. Hall, "Electron-Hole Recombination in Germanium," Phys. Rev., vol. 87, no. 2, p. 387, 1952.

[3] S. Rein, T. Rehrl, W. Warta, and S. W. Glunz, "Lifetime spectroscopy for defect characterization: Systematic analysis of the possibilities and restrictions," J. Appl. Phys., vol. 91, no. 3, pp. 2059-2070, 2002.

[4] R. E. Brandt, R. C. Kurchin, V. Steinmann, D. Kitchaev, C. Roat, S. Levcenco, G. Ceder, T. Unold, and T. Buonassisi, "Rapid semiconductor device characterization through Bayesian parameter estimation," Joule, vol. 1, no. 4, pp. 843-856, 2017.

[5] V. Steinmann R. Jaramillo, K. Hartman, R. Chakraborty, R. E. Brandt, J. Poindexter, Y. S. Lee, L. Sun, A. Polizzotti, H. H. Park, R. G. Gordon, and T. Buonassisi, "3.88\% Efficient Tin Sulfide Solar Cells using Congruent Thermal Evaporation," Adv. Mater., vol. 26, no. 44, pp. 7488-7492, 2014.

[6] M. Burgelman, P. Nollet, and S. Degrave, "Modelling polycrystalline semiconductor solar cells," Thin Solid Films, vol. 361-362, pp. 527-532, 2000.

[7] N. M. Mangan, R. E. Brandt, V. Steinmann, R. Jaramillo, C. Yang, J. R. Poindexter, R. Chakraborty, H. H. Park, X. Zhao, R. G. Gordon, and T. Buonassisi, "Framework to predict optimal buffer layer pairing for thin film solar cell absorbers: A case study for tin sulfide/zinc oxysulfide," $J$. Appl. Phys., vol. 118, no. 11, p. 115102, 2015.

[8] A. A. Istratov, H. Hieslmair, and E. R. Weber, "Iron and its complexes in silicon," Appl. Phys. A, vol. 44, pp. 1344, 1999.

[9] V. Vähänissi, A. Haarahiltunen, H. Talvitie, M. Yli-koski, and $\mathrm{H}$. Savin, "Impact of phosphorus gettering parameters and initial iron level on silicon solar cell properties,"

Progress in Photovoltaics, vol. 21 no. 5, pp. 1127-1135, 2012.

[10]M. A. Green, Solar cells: operating principles, technology, and system applications. Englewood, NJ: Prentice-Hall, 1982.

[11]D. T. Rover, P. A. Basore, and G. M. Thorson, "Solar cell modeling on personal computers," in IEEE photovoltaic specialists conference, 1985 .

[12] H. Haug and J. Greulich, "PClDmod 6. 2 - Improved simulation of $\mathrm{c}-\mathrm{Si}$ devices with updates on device physics and user interface," Energy Procedia, vol. 92, no. 1876, pp. 60-68, 2016.

[13] G. Zoth, W. Bergholz, G. Zoth, and W. Bergholz, "A fast, preparation-free method to detect iron in silicon," J. Appl. Phys, vol. 67, no. 11, pp. 6764-6771, 1990.

[14]B. Feichtinger, J. Waltl, and A. Gschwandtner, "Localization of the Fe0-Level in Silicon," Solid State Commun., vol. 27, pp. 867-871, 1978. 